\title{
Composition of Wireless Sensor Module for Vanet Applications
}

\author{
Aayushi Patel ${ }^{1}$, Shilpa $G^{2}$, Vidyashree $\mathbf{P ~ N}^{3}$, Thangadurai $\mathbf{N}^{4 *}$ \\ ${ }^{1,2,3}$ UG Scholar, ${ }^{4}$ Associate Professor and Head of the Department, \\ Department of Electronics Communication and Engineering \\ School of Engineering and Technology, Jain University, Bangalore, India \\ *Corresponding Author: ${ }^{4}$ mrgoldjain2015@gmail.com
}

\begin{abstract}
In Vehicular Ad-hoc Network (VANET) field enormous research is done recently. VANET consists of dynamic nodes in larger numbers which are mobile nodes over an area. These VANET applications should be thoroughly verified before deployment. To test these technologies in real time is not feasible which involves lot of safety issues; various reports of the testing can't be generated. To overcome this limitation can carry out simulation of VANET in the virtual environment. The network simulation is gift in this research environment. Simulation of VANET is divided into two categories (i) Traffic simulation which generates of traffic movement, defines the mobility model for vehicle also creating traffic movement and (ii) Network simulation generates communication protocols by inter communicating vehicle.
\end{abstract}

Keywords - VANET, MANET, Network simulators, Traffic simulation, ARDUINO.

\section{Introduction}

\section{A. VANET}

Vehicular Ad-hoc Networks (VANETs) are a form of mobile adnetwork (MANETs). VANETs were first said and exhibited in 2001 under "auto to-auto specially appointed portable correspondence and systems administration" applications. VANET is utilized to provide interchanges among adjoining vehicles, adjoining vehicles and closest ensconce roadside substructure. VANETs are a vital piece of the wise transportation frameworks (ITS) structure. Now and then, VANETs are eluded as Intelligent Transportation Networks and fundamental VANET design is appeared in Fig.1.

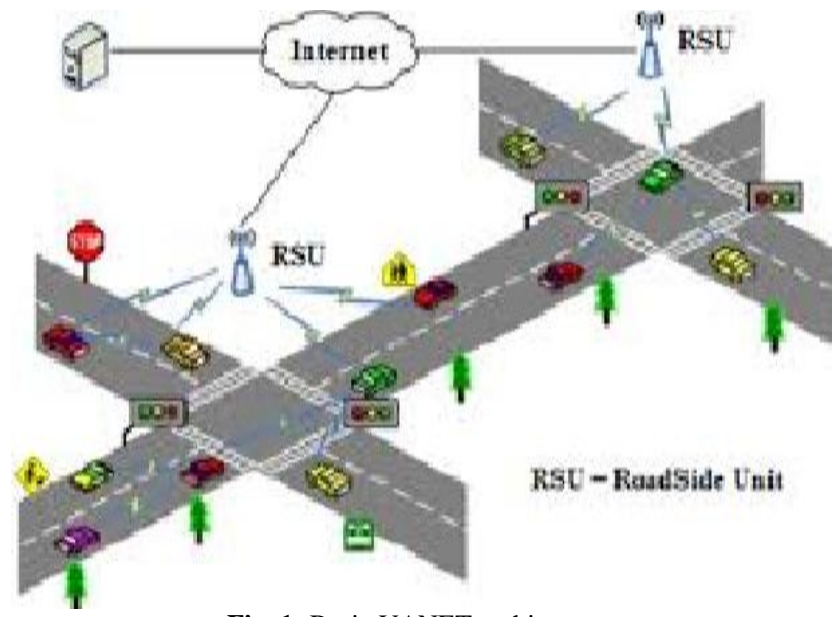

Fig. 1: Basic VANET architecture
VANETs take after the IEEE 802.11p gauges assigned to Wireless Ad Hoc Vehicular Environment (WAVE). While, in the mid 2000s, VANETs were an essential coordinated usage of MANET

standards, they have from that point forward cutting edge into a field of research in their own particular right [9]. By 2015, the expression VANET bring out to be generally synonymous with the more particular term between vehicle correspondences (IVC), anyway the point of convergence remains on the part of wilful voluntary networking, comparatively less on the utilization of infrastructure system like Road Side Units (RSUs) or cell systems.

\section{B. Applications}

- $\quad$ Electronic brake lights, security attacks, safety purposes.

- Platooning which grants vehicles to firmly (down to a couple of inches) take after a main vehicle by remotely getting quickening and controlling data, accordingly shaping electronically coupled "street trains".

- Traffic data frameworks, which utilizes VANET correspondence to present up-to-the moment hindrance records to a vehicle's satellite route framework.

- $\quad$ Road Transportation Emergency Services where VANET correspondences, systems, and street security cautioning are utilized to diminish deferrals and accelerate crisis save activities to spare the lives of those harmed.

On-The-Road Services it is likewise imagined that future transportation parkway would be one that is "data driven" and "remotely empowered". When one drives out and about, VANETs can help the driver to find administrations (shops, service stations, and so forth) on that road. Drivers can find the nearest destination they are looking for. 


\section{Wireless Sensor Networks}

Wireless sensor modules are the spatially conveyed independent sensors to screen physical environmental circumstances, for example, temperature, sound, weight, moistness and away from the starting point and to mutually get their information had come through the system to a principle area. In addition to that recent systems are bi-directional, additionally empowering control of sensor action. The advancement of remote sensor modules for systems that are utilized as a part of numerous mechanical and buyer applications, it's like modern process observing and control, machine wellbeing checking, and so forth. These modules are used in many applications like area monitoring, weather monitoring, healthcare monitoring, etc. Here we are using integrated module for various applications. [10]

\section{Literature Survey}

U.Subhadra Anjani (2015) proposed a utilization of Mobile Agent in VANET for estimating natural information by utilizing RISC processor which comprises of 3 noteworthy fragments: the sensors, the microcontroller, and the ethernet chip which is utilize LAN. It involves the three originations embedded frameworks, TCP/IP correspondence, and a climate station. It gives constant information of climate in remote/difficult to reach areas through a remote/wired association. [2]

S. M. Nadim Uddin (2016) composed a cognitive radio empowered VANET for multi-specialist based traffic activity administration framework; it shows an intellectual radio empowered VANET for multi-operator based keen movement of traffic framework. The proposed model has two distinct information exchange system layouts, namely dynamic module (vehicle to vehicle communication using cognitive radio) and semi-dynamic module (vehicle to Road-Side-Unit) [1].

Omar Alfandi (2016) methodology utilizing wireless sensor networks for calculating the speed of vehicles. This formula is used to calculate the speed of a vehicle using wireless sensor networks proved to be effective and reasonably reliable as shown in the results of the testing. The results show that the method proved to be reliable and close to the real velocity, and no linear correlation in difference of real and measured velocity shows that the speed measurement is free of systematic errors. [3]

Vinh Hoa (2014) gave a study of security attacks and solutions in VANET dangers occurred by security assaults are one of the significant security issues for the VANETs that are compelling the sending of the vehicular specially ad hoc systems [11]. They exhibited an upto-date gathering of assaults harming VANETs, inspected the down to earth situations, talked about the current solutions for manage attacks, and portrayed each assault to have an exhaustive investigate it. [4]

Neha G. Gupta (2017) did a real time implementation of vehicular ad-hoc network and has developed a vehicular ad-hoc environment by proposing a network vehicle model using microcontroller, sensors and communication technology. V-2 V trade of status data happen, where LCD installed in every one of the vehicles indicates speed, position, and separation between each other. Additionally one use of VANET is played out that in mischance location/evasion to demonstrates the system availability of models, to precisely portray impact of vehicle portability on VANETS. [5]

Prabhakar D. Dorge (2016), Implemented MIMO and AMC Techniques in WiMAX Network based VANET System. The outlined VANET framework demonstrates that the general execution of the AOMDV directing convention is superior to anything other three steering conventions regarding nature of administration parameters [6]. The DSDV steering convention gives bring down result as far as throughput, most extreme postponement, greatest jitter, and bundle conveyance proportion and parcel misfortune proportion than other directing conventions. The explanation for is that DSDV is the proactive directing convention. This sort of conventions keeps up new arrangements of goals and their courses by occasionally conveying steering tables all through the system.

Farhan Aadil (2012) gave a concept of VANET- based Warning Generation System using Cellular Networks, GPS, and Passive RFID Tags. It proposes the integration of EPTs for automatic vehicle identification in an RFID-based traceability system. For this implementation, each EPT will have a reading point combination called an RSU, and each vehicle will also have an RFID tag. The reader will record information from each vehicle's RFID and forward it to the control room. Cell phones are also used to automatically detect vehicle locations. By integrating these RFIDs on EPTs and simulating them, the system is now fully automatic - road operators can view the traffic from their control room with minimum investment. This system is based on the service platform for the mobile and e-tolling system called METS. [7]

Hua Qin, Zi Li (2016) designed an Integrated Network of Roadside Sensors and Vehicles for Driving Safety. To get the better from a pure VANET, an integrated VANET-WSN system was proposed for efficient vehicle-sensor and sensor-sensor interaction. [8]

\section{A. Limitations of the Current Work}

- The work is feasible with only few of the nodes.

- The fault tolerance of the system is more i.e., the nodes fail anytime because of hardware meddling and software snag.

- $\quad$ No mobility model to upgrade the current strategy.

- All the modules required are not integrated they are analysed separately.

- Does not have GPS module in order to track the average performance of the vehicle.

\section{B. Problem Definition}

The analysis of few models is concise to only 4-5 nodes (vehicles) .The performance of the system is hypothetical that is multipath propagation. Faults occur anytime when hardware or the software goes wrong. The delay will be increased and information will be loss when the routing signals obtained from a faulty vehicle.

\section{Objective}

- The number of nodes that can be linked can be extended to ' $n$ ' nodes to a certain extent. Developing a mobility model in order to improve the representation of the system for real time analysis. Based on the vehicles, buildings, roads, maps, vehicular density and driver's behaviour an mobility model can be plan out.

- The fault can be minimised to an extent by reducing the hardware tampering and reducing the software faults in a system which can be defend the network from these faults.

- GPS module can be added in order to track the location of the vehicle. Execution of distributed computing ideas can give benefits in programming, equipment and platform level. The capacity can likewise be utilized as an administration since cars have terabytes of memory. By utilizing cloud, numerous applications are anticipated like sight and multimedia administrations, information conveyance, area sharing, eapplications, P2P administrations (Peer-to-Peer).

\section{Methodology}

\section{A. Hardware Requirements}

- The on-board sensors are available in the vehicle are utilized to discover vehicle's area, speed and development.

- Another imported perspective for VANET moving toward the vehicle is about the speed at which its neighbouring vehicles are towards to it. The RF transmitting circuit comprise of a RF 
transmitter module interfaced. It is a low power, superior CMOS microcontroller with programmable Flash memory in constructed.

- Infrared sensor is utilized with engine drive; to pivot the wheel of vehicle focus shaft engine is utilized. As the wheel finish one turn, IR sensor joined to it will radiate light from IR transmitter and IR recipient gets it. For one pivot speed is figured as transformation per sec. This speed esteem is partitions by 60 for every min revolution per min (RPM). Cycles every moment, is measure of the recurrence of a turn.

- Other sensors are used to monitor the physical parameters such as temperature, pressure, humidity as shown in block diagram Fig.2.

- In Vehicular Ad-hoc Network (VANET), confinement is turning into a basic need since numerous VANET applications constrain position information. The utilization of GPS unit inside the vehicle permits the vehicle position by discovering its area arranges.

\section{B. Software Requirements}

- $\quad$ Sending nodes: These are the main target of the project. A wireless mote is attached to the vehicles or moving objects. It will be constantly sending dummy packets, which also contain the nodes ID to be processed by the fixed nodes.

- $\quad$ Fixed node: Its function is to receive data, i.e. sense the moving motes and receive their ID and send it to the Base Station. Only the strongest signals are chosen to be sent to the Base Station together with the ID and time of receipt. These motes are scattered across the lane and have a fixed distance between them. This allows for the calculation of the speed of the vehicle in a portion of the road, between two fixed nodes.

- $\quad$ Base station: The base station gathers the data from the fixed points and makes decisions based on the first packet having the signal strength of the moving mote. Using this information, a graphical interface is deployed to calculate the vehicle speed by filtering the data received and finally displaying the results.

- $\quad$ Sending packets: The mobile sending motes are the representation of the vehicles in a highway.

- $\quad$ Receiving packets: Fixed motes are scattered across the lane or path which the mobile nodes pass by.

- $\quad$ The data is received and gathered for the further analysis and calculation purpose

- $\quad$ The speed can be evaluated by the simple relation is $\mathrm{v}=\mathrm{d} / \mathrm{t}$.
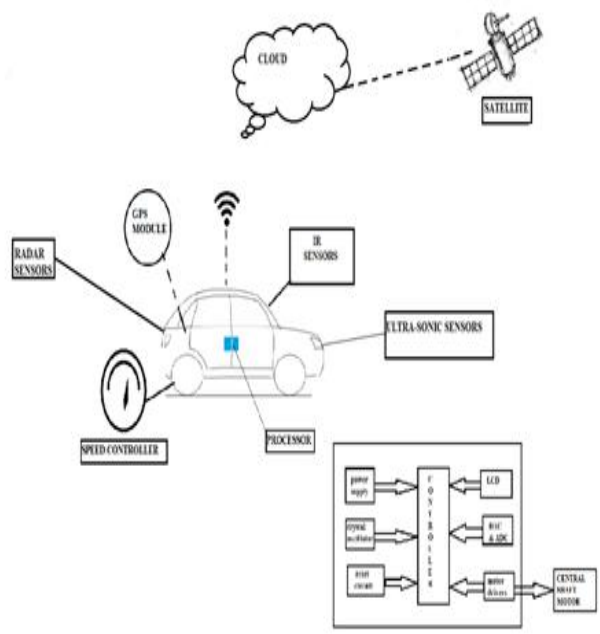

Fig. 2: Proposed block diagram

\section{Arduino Uno R3}

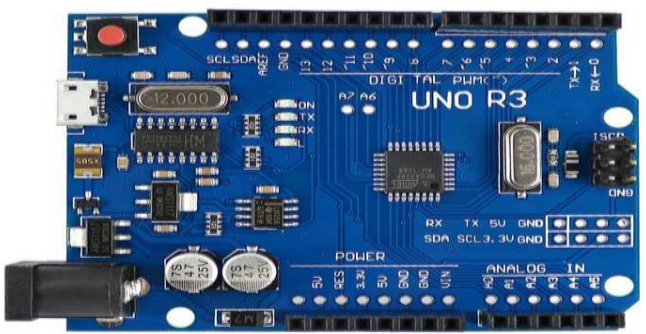

Fig. 3: Arduino Uno Microcontroller

Technical specifications of ATmega328 detailed in Table 1 which consists of 14 digital input/output pins (out of which six can be utilized as PWM outputs), a power jack, a $16 \mathrm{MHz}$ ceramic resonator, along with USB connector to processor to system, 6 analog inputs, an ICSP header, and a reset pin. It contains everything expected to help the microcontroller; necessarily collaborate with a PC and a USB link or by using AC-to-DC connector to power the battery.

Update 3 of the board has the accompanying new highlights:

- 1.0 pinout: included SDA and SCL pins that are close to the AREF spin and two other new pins set close to the RESET, the IOREF that enable the shields to adjust to the voltage gave from the board.

- $\quad$ Stronger RESET circuit.

- Atmega 16U2 supplants the 8U2.

\section{Sensors with Circuit Diagrams}

\section{Temperature and humidity Sensor}

Calibrated digital output can be obtained by DHT11 interfaced with any microcontroller like Arduino, Raspberry $\mathrm{Pi}$, etc and obtain near real time results (Fig.4). It is cost effective which established very high reliability along with durability.

The DHT11 is a fundamental computerized temperature and humidity sensor. It utilizes a capacitive sensor and a thermistor to quantify the encompassing air, and parts out an advanced flag on the information pin where there no analog information pins required. It is genuinely easy to utilize, yet requires watchful planning to get information. The main genuine constraint of this sensor is just get new information from it once like clockwork, so when utilizing our library, sensor capturing up to 2 seconds old. Highlights are I/O of 3 to $5 \mathrm{~V}$ power, $2.5 \mathrm{~mA}$ max current utilize amid transformation (while asking for information), for $20-80 \%$ dampness readings within $5 \%$ exactness followed by $0-50^{\circ} \mathrm{C}$ temperature readings with \pm $2^{\circ} \mathrm{C}$ precision, and $1 \mathrm{~Hz}$ sampling rate (once consistently).

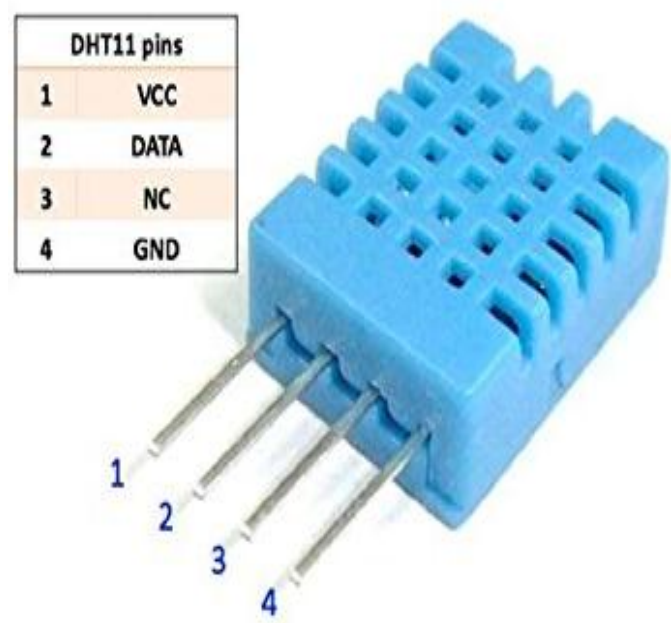

Fig. 4: Temperature and humidity sensor 


\section{Rain Sensor}

A sensor used during rain enhances the safety and comfort while driving, as they avoid the need of a driver to alter the wiper setting on the windshield. This ensures clear view via the windshield without wiper blades being rattled. When water falls on the windshield these sensors are automatically switched on the wiper (Fig.5).

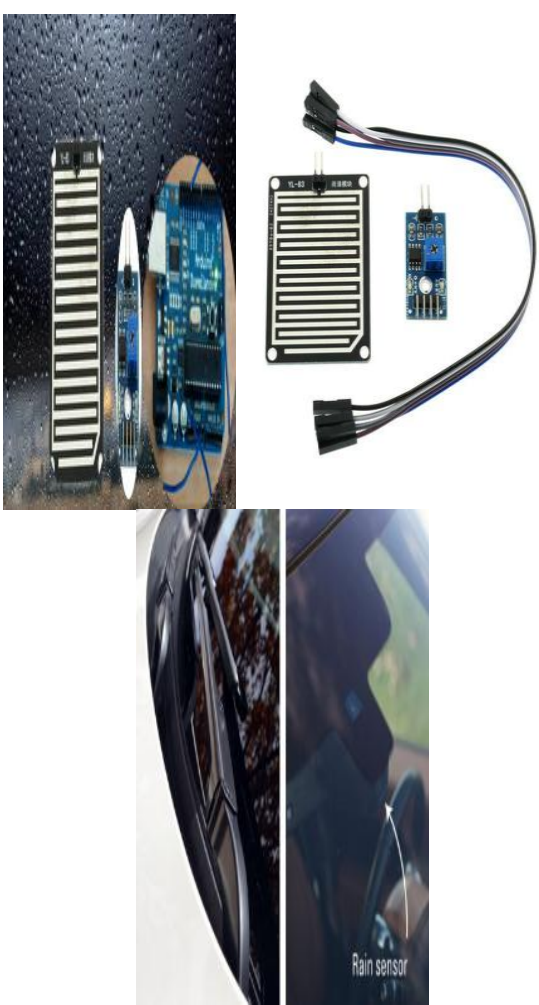

Fig. 5: Rain sensor

When water is down-pouring, the car's sensors estimates and gauges the rain drops that are accumulated above the windshield. The inside reflected light by the windshield glass is recognized by the sensors, then only few light will be reflected to sensor when there is more than expected raindrops on the windshield. Here's how your car windshield rain sensors works: The amount of rain on the car windshield is sensed by the vehicle, and accordingly speeds up the wipers of the windshield and detects the amount the rain. The sensor is arranged on an uncommon section behind the vehicle's back view reflect and wired through the rooftop.

\section{Piezoelectric sensor}

Piezoelectric sensor is a transducer which converts stress applied on it into some electrical energy (Fig.6).

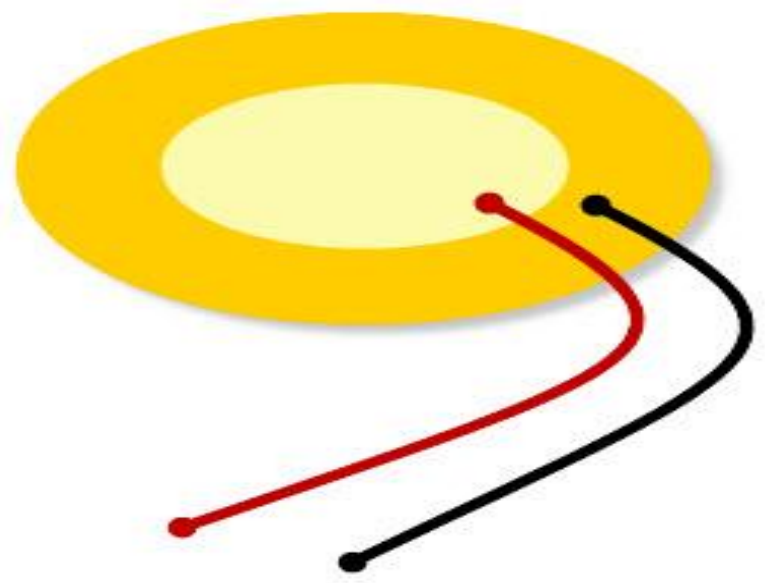

Fig. 6: Piezoelectric sensor
It is simply uses the principle of energy conversion from one form to another i.e., it converts physical stress into electrical energy. Stress can be any of power, weight, speeding up and touching potential. Because these are all kind of stresses and piezoelectric sensor converts such kind of physical forces into electrical form. Piezoelectric pressure transducer working principle

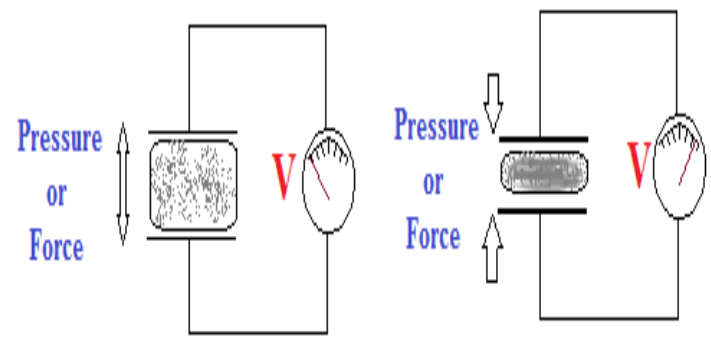

Fig. 7: Piezoelectric pressure transducer working principle

Piezo sensors are established by piezo element, these utilize the piezo electric effect which converts Pressure, Force, Strain, and Temperature (sometimes) into Electrical current. Between the two metal plates a piezo element is placed for both the buzzer and transducer. Fig.7 explains the working principle of piezo element and it can be easily calculated by voltmeter when the pressure executes electric charge originate in piezo elements. The ground terminal is connected to GND and Arduino Analog input pin A0 connected to positive signal out terminal of sensor which will be able to create high sensitivity vibration monitoring equipment. And also wireless vibration monitoring device can be developed by interfacing with radio frequency wireless transceiver modules. A potential difference of same magnitude of force is produced across piezoelectric transducer when stress is given to the piezo electric sensors. So it can be used to convert mechanical energy into electrical energy easily. Piezoelectric sensors produce analog output voltage.

\section{PIR sensor}

Passive IR (PIR) sensor is utilized to measure the infrared (IR) radiation which is being discharged through objects in its field of view (Fig.8). These are often mostly used in PIRbased motion detectors. When the object temperature is varies from normal to absolute zero temperature then it emits heat energy will be in the form of radiation. The human eye cannot view this radiation because it radiates at IR wavelengths, but by using electronic devices these radiations can be detected which are exclusively developed. The expression "passive" alludes to the way that PIR gadgets don't transmit or produce vitality for recognition purposes. They work completely by distinguishing IR radiation reflected or discharged from objects, but they don't recognize or measure "warmness".

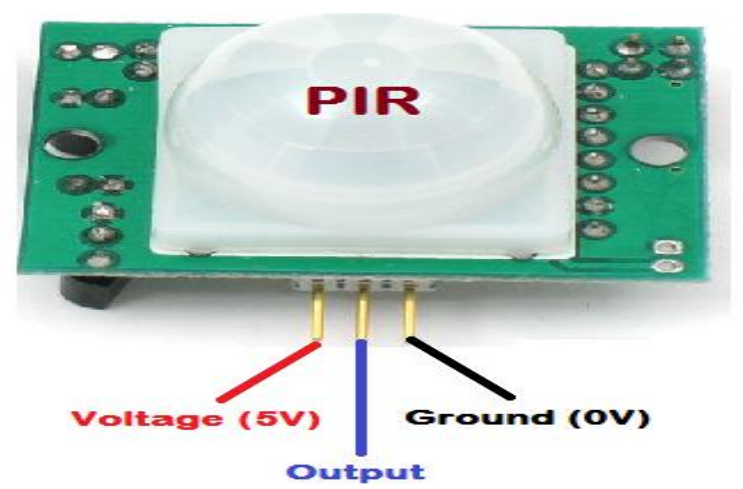

Fig. 8: PIR sensor 


\section{X2 LCD Display}

Calculators, digital watches, television sets, computers, mobile phones, and traffic signal lights use some kind of display is known as LCD display which is an electronic display module consists of liquid crystal to generate a perceptible image. The $16 \times 2$ LCD which generates 2 lines display consists of 16 characters per line (Fig 9).

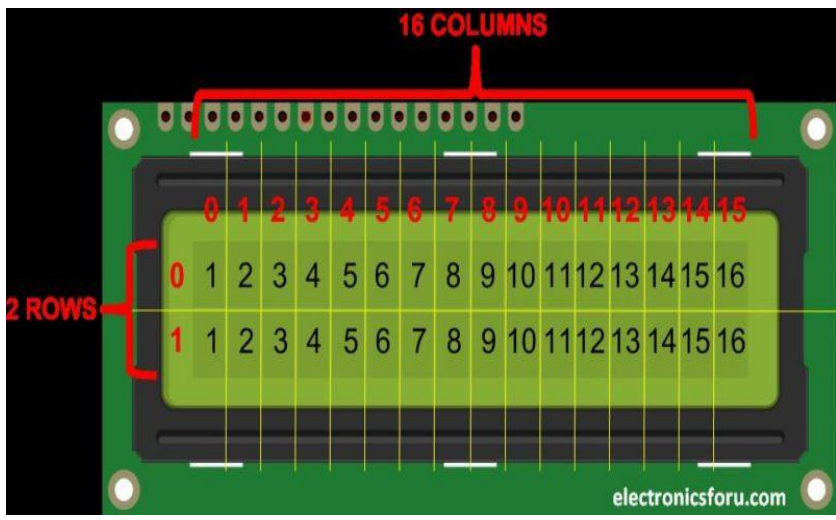

Fig. 9: 16X2 LCD pinout diagram

\section{LED \& Buzzer}

A P-N junction diode emits light when it is activated and it usually requires a small voltage for its operation. And is semiconductor light source which has two terminals and is known as LED. When voltage is applied, the electrons will recombine with the holes and this recombination will release energy in the form of light.

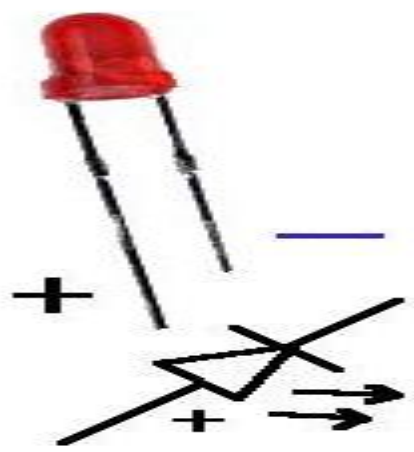

Fig. 11: LED

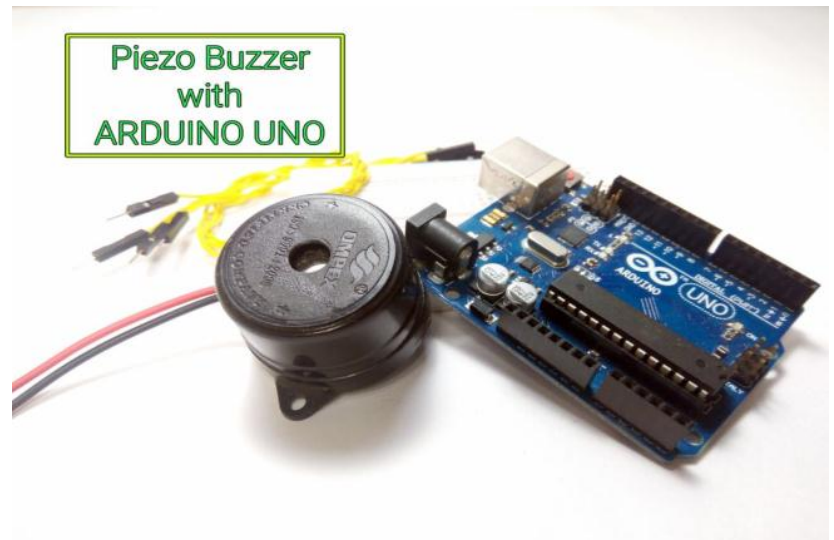

Fig. 12: Buzzer

Piezo buzzers are used for making beeps alarms sounds and tones (Fig.12). They can be used in alarm systems, for keypad feedback, or some games, etc. Light weight, simple construction and low price make it usable in various applications like car/truck reversing indicator, computers, and call bells watches etc.

\section{Vanet Composition}

\section{A. Algorithm}

When the information sends the data through packets, router will discovered path and assign which path has been chosen. Once the path is assign create a bi-directional link node with a $10 \mathrm{Mbps}$ capacity. The output will be encoded and decrypted before performing the final evaluation (Fig.13). Figure 14 shows the assigned path flow between the nodes, packet sending and corresponding simulation results shown in Figure 15 and 16.

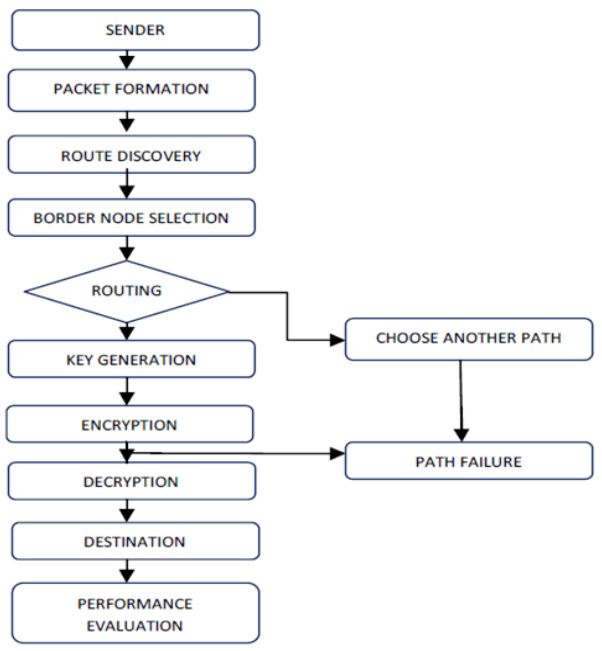

Fig. 13: Proposed algorithm

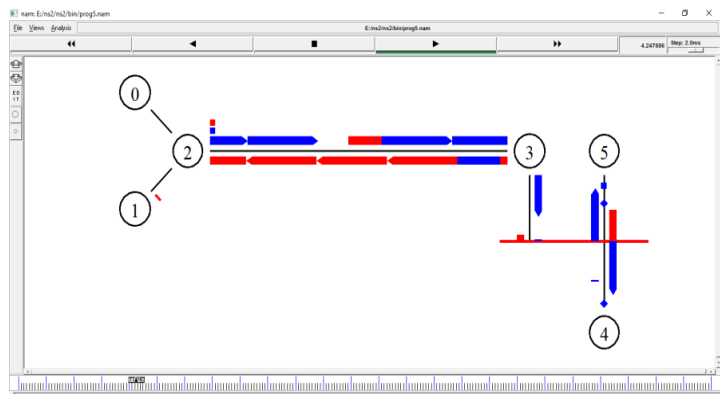

Fig. 14: Example for defining nodes, links, queues and topology

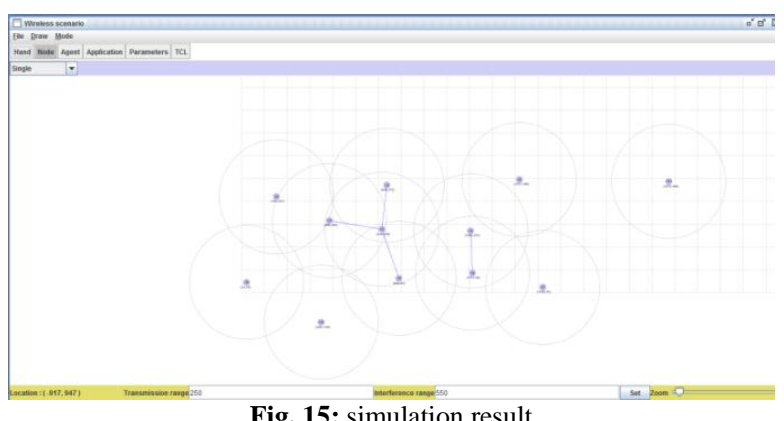

Fig. 15: simulation result

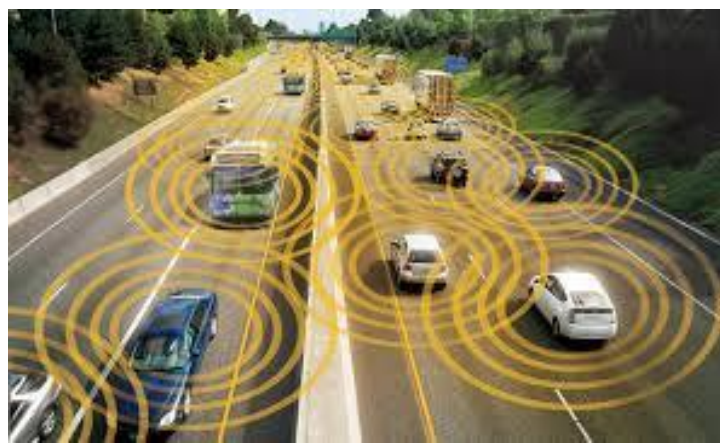

Fig. 16: practical illustration of Interconnection of nodes (wireless) 


\section{B. Construction \& Operation}

Sensor confront is commonly defined as when the IR radiation transmit through the front of the sensor where the center of an PIR sensor is in strong state sensor or many number of sensors, produced using pyro-electric materials which when presented to warm create vitality. Gallium Nitride $(\mathrm{GaN})$, Caesium Nitrate (CsNO3), polyvinyl fluorides, subsidiaries of phenylpyridine, and cobalt phthalocyanine are the materials utilized as a part of PIR sensors.

Every last PIR sensor recognizes changes in the measure of infrared radiation which is affecting it, which mainly relies upon the temperature and surface attributes of the object kept before the sensor. For example, a creature or people go before the foundation, for example, a divider wall, the temperature by then in the sensor's field of view will ascend from room temperature to body temperature, and afterward come back once more to normal condition. The recognition took place when the sensor changes over the subsequent in approaching IR radiation into an adjustment in the yield voltage. The detector can also trigger the objects of proportionate temperature yet extraordinary surface attributes may likewise have an alternate IR discharge pattern example, and subsequently moving them concerning the foundation.

\section{Results and Discussion}

After completing the procedure discussed can run the simulation using Network Simulator. The information sharing between two or more static nodes representation is shown in Fig. 17 and Fig. 18 shows the mutual sharing of concept between the nodes- 1 .

Fig. 19 shows OBU (on board unit) in vehicular networks which realises V2V communication. Boundary around each node indicates the communication range of a node, the message passed to neighbouring OBU nodes.

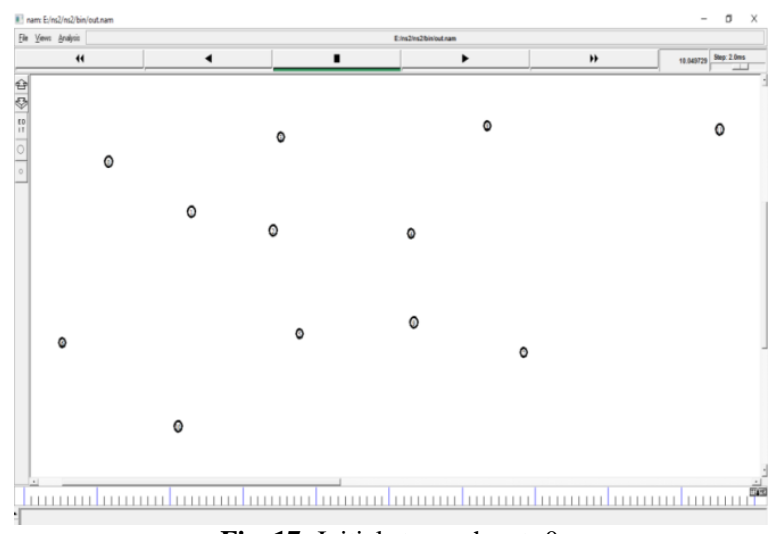

Fig. 17: Initial stage when $t=0$

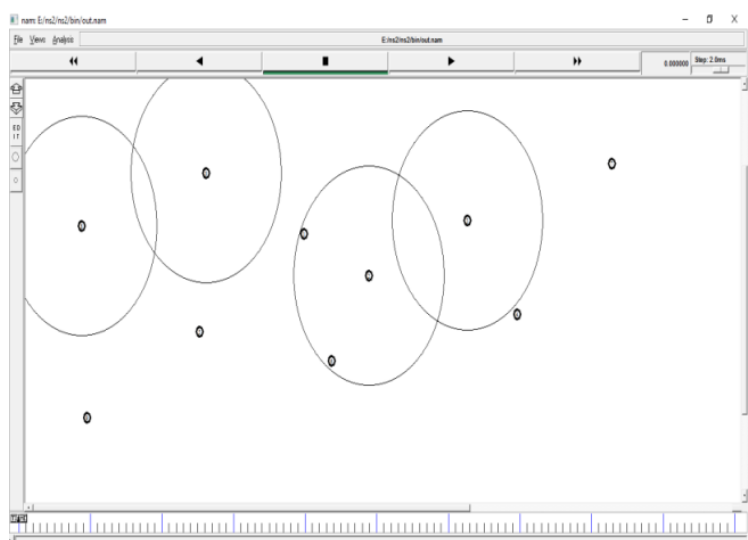

Fig. 18: Mutual sharing of information between the nodes-1

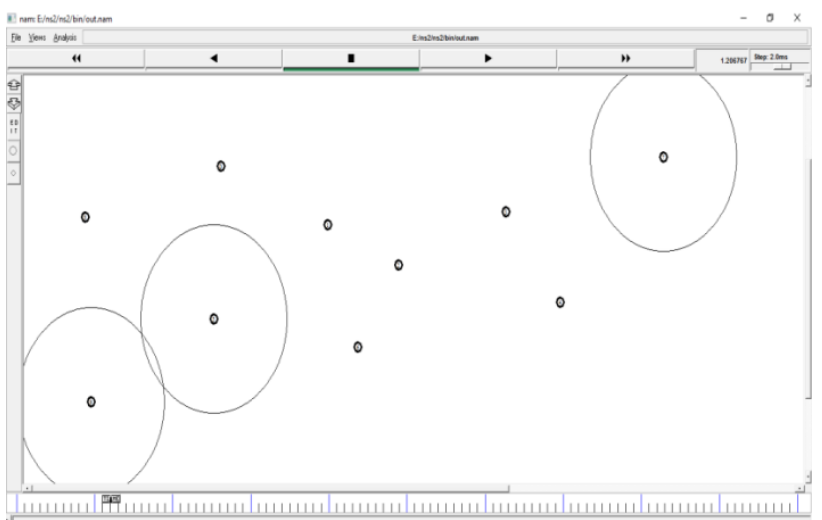

Fig. 19: Mutual sharing of information between the nodes-2

The behaviour or performance of VANET in different scenarios measured or performance metrics considered, in terms of average packet delivery ratio, average throughput and average end to end delay. The packet delivery ratio (PDR) is defined as the ratio of number of data packets transmitted to the destination successfully and is calculated using mathematical representation shown in Fig. 20 utilizing the relation of PDR $\mathrm{PDR}=\sum \mathrm{Pr} / \sum \mathrm{Ps}$

Where Ps is total number of packets sent,

$\operatorname{Pr}$ is total number of packets received successfully.

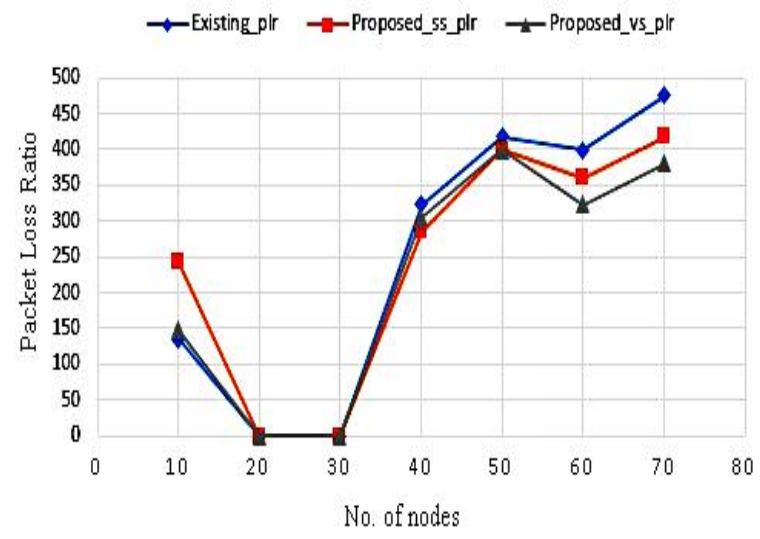

Fig. 20: Performance of VANET

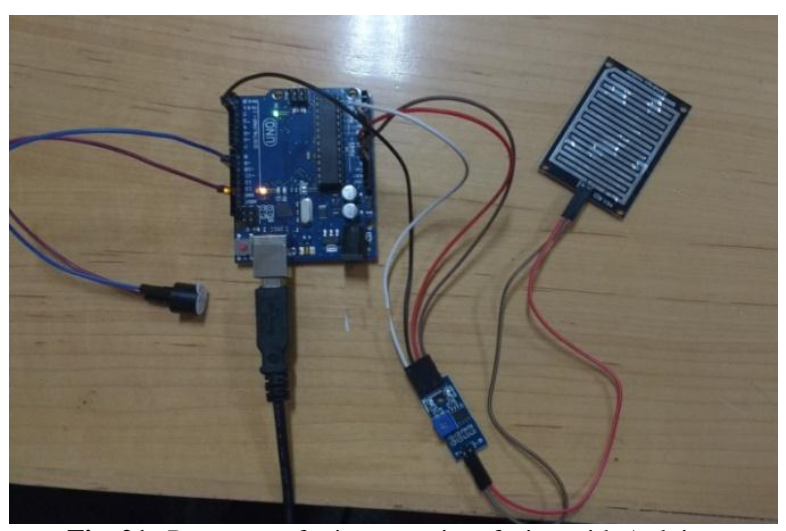

Fig. 21: Prototype of rain sensor interfacing with Arduino

Arduino (A0) pin is connected with analog output terminal, and the output will be taken from digital pin D10. The $5 \mathrm{~V}$ and ground power source applied to the rain sensor module, when the rain falls on the analog output varies from $5 \mathrm{~V}$ to 0 . If the sensor condition remains Dry then output will be $5 \mathrm{~V}$, and this output voltage range using single transistor if it's required (Fig. 21). Rain sensor and buzzer are connected to arduino, in this experiment buzzer device used as an alarm.

Figure 22 shows the hardware connections of Piezoelectric sensor interfacing with Arduino. Piezoelectric sensors have two output pins and one is positive potential (means vdd) and other is at negative potential (means ground). Positive potential pin is 
connected to pin 3 analog channels and negative potential pin is connected to ground. For protection purpose a resistor of one mega ohm is connected between them. A led is connected to pin 13 of arduino to check working of sensor output.
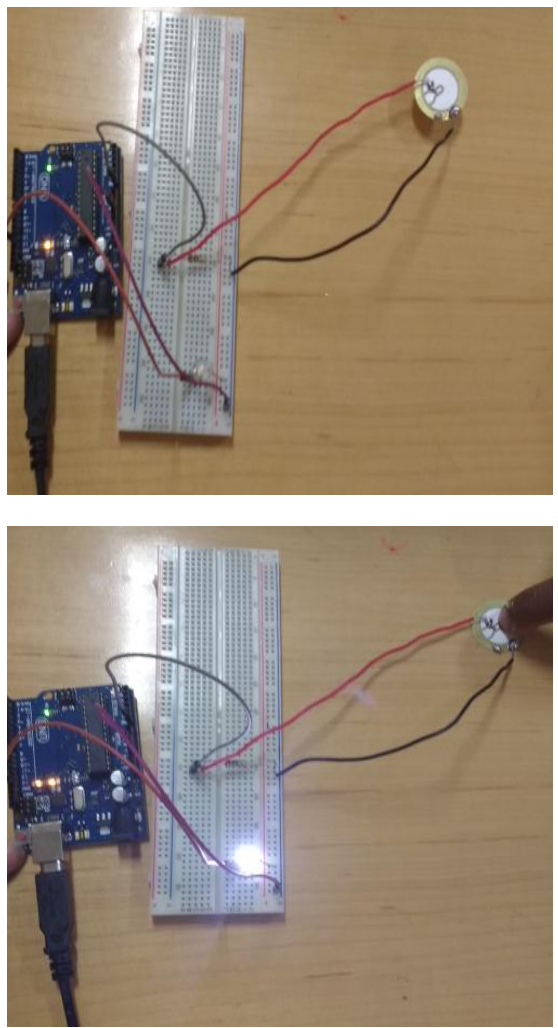

Fig. 22: Prototype of Piezoelectric sensor interfacing with Arduino
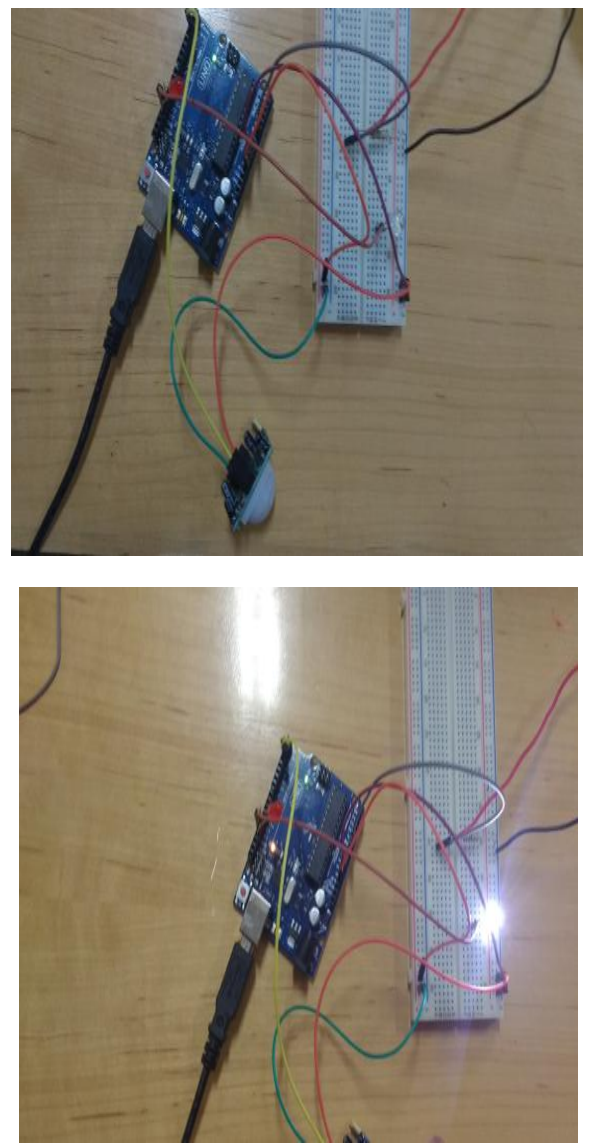

Fig. 23: Prototype of PIR sensor interfacing with Arduino

PIR sensor has two slots which are sensitive to infrared energy. If the sensor is in normal condition, both the slots measure the same amount of energy radiated by walls. If a warm body crosses then first cross PIR sensors, which produce positive differential change between two slots. If an animal or human being leaves the sensing area, a respective negative differential change is produced. The changes in PIR sensor can detect the motion of different objects by its infrared energy radiation.

\section{Conclusion}

WSNs are possible today due to technological advancements in various domains envisaged to become a crucial part of all applications. Designed constraints are to be satisfied for realisation of sensor networks. NS-2 can be used as one of the simulation tools to study the network behaviour. Simulation of vehicular networks is done by using network simulation in NS-2. Here we can see that all the static nodes can share information within the defined radius. And cannot communicate with the nodes which are out of assigned radius and is applied to nodes within few metres of range. The nodes are defined using two dimension with z-axis being zero. The node values can be used in calculating the distance between them by Using distance formula.

\section{References}

[1] Farhan Aadil, Zeshan Iqbal and Adeel Akram, "Smart Computing Review Implementation of VANET-based Warning Generation System using Cellular Networks", Smart Computing Review, vol. 2, no. 6, pp. 391-400, 2012.

[2] Hua Qin, Zi Li, Yanfei Wang, Xuejia Lu and Wensheng Zhang, Guiling Wang, "An Integrated Network of Roadside Sensors and Vehicles for Driving Safety Concept, Design and Experiment," IEEE International Conference on Pervasive Computing and Communications (PerCom), pp. 79-87, 2010, Mannheim.

[3] Narendra Mohan Mittal and Savita Choudhary, "Comparative Study of Simulators for Vehicular Ad-hoc Networks (VANETs)", International Journal of Emerging Technology and Advanced Engineering, Vol.4, Iss. 4, pp. 528-537, 2014.

[4] Neha G.Gupta, Rajesh D.Thakre, Yogesh A. Suryawanshi, "Real Time Implementation of Vehicular Ad-hoc Network," International Journal of Engineering Trends and Technology, Vol. 45, No. 3, 2017.

[5] Omar Alfandi, Arne Bochem, Mehdi Akbari Gurabi, Alberto Rivera Dias, Md.Istiak Mehedi, Dieter Hogrefe, "Calculating the Speed of Vehicles Using Wireless Sensor Networks," 2016 Federated Conference on Computer Science and Information Systems (FedCSIS), pp. 1043-1047, Gdansk, 2016

[6] Prabhakar D. Dorge, Dr. Sanjay S. Dorle and Megha B.C, "Implementation of MIMO and AMC Techniques in WiMAX Network based VANET System," International Journal of Information Technology and Computer Science, vol. 2, pp.60-68, 2016.

[7] S. M. Nadim Uddin, Nafees Mansoor, Sazzad Hossain, "Cognitive Radio Enabled VANET for Multi-agent Based Intelligent Traffic Management System," 1st International Conference on Advanced Information and Communication Technology, Bangladesh, 2016.

[8] Saha A. K. and Johnson D.B., "Modeling the mobility for vehicular ad hoc networks(VANET)," in Proceedings of ACM International Workshop on Vehicular Ad Hoc Networks, pp. 91-96, 2004

[9] U Subhadra Anjani, S Mohammed Rafi,Y Kiran, "Application of Mobile Agent in VANET for Measuring Environmental Data by Using RISC Processor," International Journal of Emerging Engineering Research and Technology, vol. 3, 2015.

[10] N Thangadurai, R Dhanasekaran, "Energy Efficient Cluster based Routing Protocol for Wireless Sensor Networks" International Journal of Computer Applications, Vol. 71 Iss.7 pp.43-48, 2013.

[11] Vinh Hoa LA, Ana Cavalli, "Security Attacks and Solutions In Vehicular Ad Hoc Networks: A Survey," International Journal on AdHoc Networking Systems, Vol. 4, No. 2, 2014. 